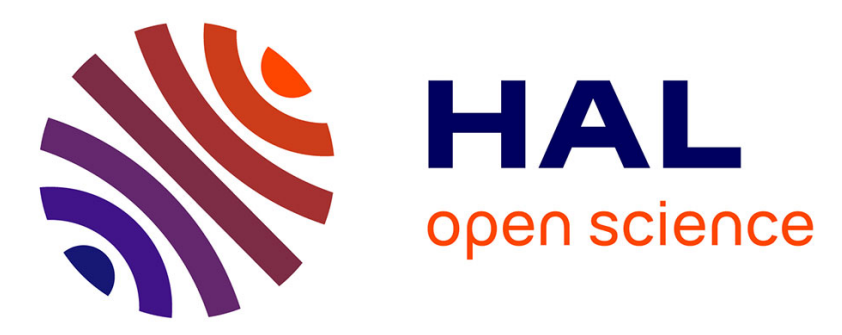

\title{
Inherent limitations on disjoint-access parallel implementations of transactional memory
}

Hagit Attiya, Eshcar Hillel, Alessia Milani

\section{To cite this version:}

Hagit Attiya, Eshcar Hillel, Alessia Milani. Inherent limitations on disjoint-access parallel implementations of transactional memory. SPAA, 2009, Unknown, pp.69-78, 10.1145/1583991.1584015 . hal-00992693

\section{HAL Id: hal-00992693 \\ https://hal.inria.fr/hal-00992693}

Submitted on 19 May 2014

HAL is a multi-disciplinary open access archive for the deposit and dissemination of scientific research documents, whether they are published or not. The documents may come from teaching and research institutions in France or abroad, or from public or private research centers.
L'archive ouverte pluridisciplinaire HAL, est destinée au dépôt et à la diffusion de documents scientifiques de niveau recherche, publiés ou non, émanant des établissements d'enseignement et de recherche français ou étrangers, des laboratoires publics ou privés. 


\title{
Inherent Limitations on Disjoint-Access Parallel Implementations of Transactional Memory
}

\author{
(Preliminary Version)
}

\author{
Hagit Attiya \\ Department of Computer \\ Science, Technion \\ Haifa, Israel \\ hagit@cs.technion.ac.il
}

\author{
Eshcar Hillel $^{\dagger}$ \\ Department of Computer \\ Science, Technion \\ Haifa, Israel \\ eshcar@cs.technion.ac.il
}

\author{
Alessia Milani ${ }^{\ddagger}$ \\ Department of Computer \\ Science, Technion \\ Haifa, Israel \\ alessia@cs.technion.ac.il
}

\begin{abstract}
Transactional memory (TM) is a promising approach for designing concurrent data structures, and it is essential to develop better understanding of the formal properties that can be achieved by TM implementations. Two fundamental properties of TM implementations are disjoint-access parallelism, which is critical for their scalability, and the invisibility of read operations, which reduces memory contention.

This paper proves an inherent tradeoff for implementations of transactional memories: they cannot be both disjointaccess parallel and have read-only transactions that are invisible and always terminate successfully. In fact, a lower bound of $\Omega(t)$ is proved on the number of writes needed in order to implement a read-only transaction of $t$ items, which successfully terminates in a disjoint-access parallel TM implementation. The results assume strict serializability and thus hold under the assumption of opacity. It is shown how to extend the results to hold also for weaker consistency conditions, serializability and snapshot isolation.
\end{abstract}

\section{Categories and Subject Descriptors}

D.1.3 [Programming Techniques]: Concurrent Programming; F.2.2 [Analysis of Algorithms and Problems]: Nonnumerical algorithms and problems

\section{General Terms}

Theory, Algorithms, Design

\footnotetext{
*This research is partially supported by the Israel Science Foundation (grant number 953/06) and Intel Corporation.

${ }^{\dagger}$ Supported in part by a scholarship from the Israel Ministry of Science.

$\ddagger$ On leave from Sapienza, Universitá di Roma, Dipartimento di Informatica e Sistemistica, "Antonio Ruberti"; supported in part by a fellowship from the Lady Davis Foundation and by a grant MUR, FIRB Italia-Israele RBIN047MH9
}

Permission to make digital or hard copies of all or part of this work for personal or classroom use is granted without fee provided that copies are not made or distributed for profit or commercial advantage and that copies bear this notice and the full citation on the first page. To copy otherwise, to republish, to post on servers or to redistribute to lists, requires prior specific permission and/or a fee.

SPAA'09, August 11-13, 2009, Calgary, Alberta, Canada.

Copyright 2009 ACM 978-1-60558-606-9/09/08 ...\$5.00.

\section{Keywords}

Transactional memory, disjoint-access parallelism, partial snapshots, lower bound, impossibility result

\section{INTRODUCTION}

Transactional memory is an attractive paradigm for programming concurrent applications for multicores. A transaction encapsulates a sequence of operations, and it is guaranteed that if any operation takes place, they all do, and that if they do, they appear to other threads to do so atomically, as one indivisible operation. A transactional memory implementation translates high-level transaction operations to low-level primitive operations on base objects, containing the data and the meta-data needed for the implementation.

Transactional memory is seriously considered as part of software solutions and as a basis for novel hardware designs. It is therefore imperative to understand inherent tradeoffs in the design and implementation of transactional memory.

One property that is considered critical for the scalability of a transactional memory implementation is disjoint-access parallelism: operations on disconnected data should not interfere. Conceptualizing this notion is best done through the conflict graph of transactions that overlap in time. Informally, the vertices of the conflict graph correspond to data items, and there is an edge between data items if they are accessed by the same transaction. Consider, for example, four concurrent transactions: $T_{1}$ with the data set $\left\{\imath_{1}, \imath_{2}\right\}$, $T_{2}, T_{3}$, and $T_{4}$ with the data sets $\left\{\imath_{2}\right\},\left\{\imath_{3}\right\}$, and $\left\{\imath_{4}\right\}$, respectively. Figure 1 depicts the conflict graph of the execution interval of these four transactions. This conflict graph contains only one edge connecting the two vertices representing the data items in the data set of $T_{1}$ (see the formal definition in Section 2).

Several transactional memories, e.g. [4, 13], guarantee that transactions access the same base object only if their data items are connected in the conflict graph. In particular, there is no concurrent access to the shared memory by transactions that access disjoint parts of the data. In such implementations, the transactions $T_{1}, T_{3}$, and $T_{4}$ in the example above, do not access the same base object, since no path connects their data items in the conflict graph.

Another important goal is to optimize read-only transactions, i.e., transactions that access the memory only through read operations. It is desirable that in their implementations, read-only transactions do not execute primitive write 
operations to the memory, so as to reduce memory contention; implementations of read-only transactions that do not write to the memory are called invisible. Moreover, since read-only transactions do not write to data items, it seems plausible they should eventually be able to obtain a consistent view of the data, provided previous versions are kept (as is done in multi-version implementations [20, 22, 23]). Thus, read-only transactions should (eventually) terminate successfully, regardless of concurrent transactions; such transactions are called wait-free.

None of the existing transactional memory implementations is both disjoint-access parallel and has invisible, waitfree read-only transactions. Some are disjoint-access parallel and have invisible but not wait-free read-only transactions [4, 13], while others have invisible, wait-free read-only transactions but are not disjoint-access parallel [22].

Consider, for example, the four transactions above, and assume $T_{1}$ is a read-only transaction, while $T_{2}, T_{3}$, and $T_{4}$ all write to their data items. In the algorithm given in [13], which is disjoint-access parallel and has invisible read-only transactions, $T_{1}$ reads $\imath_{1}$ and $\imath_{2}$, then $T_{2}$ writes to $\imath_{2}$, finally $T_{1}$ validates its read set at commit time. The value of $\imath_{2}$ has changed since $T_{1}$ read it, and $T_{1}$ aborts. In the algorithm given in [22], which has invisible, wait-free read-only transactions, $T_{1}, T_{3}$, and $T_{4}$ all access a common counter ( $T_{1}$ reads it, while $T_{3}$ and $T_{4}$ write to it), thus violating disjoint-access parallelism.

This paper shows that there is an inherent tradeoff-no transactional memory implementation can be disjoint-access parallel and have invisible, wait-free read-only transactionsand one of these desirable properties must always be compromised. In fact, we prove a stronger result, showing that in a disjoint-access parallel transactional memory implementation with wait-free read-only transactions, a transaction reading $t$ data items must apply non-trivial primitives (e.g., writes) to at least $t-1$ base objects. Thus, a read-only transaction must perform one low-level write essentially for each item in its read set.

The wait-freedom requirement might seem too restrictive for practical purposes; however, we can prove a similar result where a read-only transaction repeatedly aborts and never terminates successfully; see further discussion in Section 6. For read-dominated applications, this implies too much wasted work.

The consistency condition commonly used for transactional memory is opacity [10]; very roughly stated, opacity requires all transactions to appear to execute sequentially in an order that agrees with the order of non-overlapping transactions. This is similar to requiring strict view serializability [21] applied to all transactions (included aborted

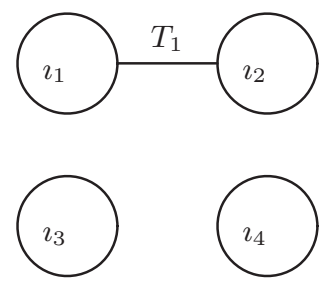

Figure 1: Example of a simple conflict graph: $\imath_{1}$ and $\imath_{2}$ are the data items of $T_{1} ; \imath_{2}, \imath_{3}$, and $\imath_{4}$ are the data items of $T_{2}, T_{3}$, and $T_{4}$, respectively. ones), extended to allow operations other than reads and writes. Our results only assume strict serializability [21], and hence hold also under the assumption of opacity. In fact, the results also hold for weaker consistency conditions, serializability and snapshot isolation.

The rest of the paper is organized as follows: Section 2 introduces basic definitions and in particular, the notion of disjoint-access parallelism. Section 3.1 presents an impossibility result showing that in a disjoint-access parallel STM implementation with invisible read-only transactions, some read-only transaction may never terminate successfully; this result is proved using only three processes. Section 3.2 strengthens this result and shows that a read-only transaction on $t$ items (in a disjoint-access parallel STM implementation with wait-free read-only transactions) must apply non-trivial primitives to $t-1$ base objects; this result requires $t+1$ processes. Section 4 extends the results to hold even with the weaker conditions of serializability and snapshot isolation. We discuss related work in Section 5, and conclude in Section 6.

\section{PRELIMINARIES}

A transaction is a sequence of operations executed by a single process on a set of data items shared with other transactions; all data items are initially 0 . We assume data items are accessed by simple read and write operations; our impossibility results clearly hold for transactional memory that also supports other operations. A complete interface of transactional memory also includes commit and abort operations, which we do not model here, since they are not needed for our impossibility results.

The collection of data items accessed by a transaction is the transaction's data set; in particular, the items written by the transaction are its write set, and the items read by the transaction are its read set. A transaction whose write set is empty, is said to be a read-only transaction. We assume the transaction's read set and write set are provided at the start of the transaction, and do not elaborate further on the manner a transaction issues its operations; this only makes our impossibility results stronger.

An implementation of software transactional memory (abbreviated $S T M$ ) provides data representation for transactions and data items using base objects, and algorithms, specified as primitive operations on the base objects, which asynchronous processes have to follow in order to execute the operations of transactions. In addition to ordinary read and write primitives, we allow arbitrary read-modify-write primitives, even those accessing several locations simultaneously. In particular, the implementation may use a cas $(o$, exp, new) that writes the value new to location $o$ if its value is equal to exp, and returns a success or failure indication.

A primitive is non-trivial if it may change the value of the object, e.g., a write or cas; otherwise, it is trivial, e.g., a read.

An event is a computation step by a process consisting of local computation and the application of a primitive to base objects, followed by a change to the process's state, according to the results of the primitive. A configuration is a complete description of the system at some point in time, i.e., the state of each process and the state of each shared base object. There is a unique initial configuration in which every process is in its initial state and every base object contains its initial value. 
An execution interval $\alpha$ is a finite or infinite alternating sequence $C_{0}, \phi_{0}, C_{1}, \phi_{1}, C_{2}, \ldots$, where $C_{k}$ is a configuration, $\phi_{k}$ is an event and the application of $\phi_{k}$ to $C_{k}$ results in $C_{k+1}$, for every $k=0,1, \ldots$. An execution is an execution interval in which $C_{0}$ is the initial configuration.

Two executions $\alpha_{1}$ and $\alpha_{2}$ are indistinguishable to a process $p$, if $p$ goes through the same sequence of state changes in $\alpha_{1}$ and in $\alpha_{2}$; in particular, this implies that it goes through the same sequence of events.

We point out that the model encompasses two levels of abstraction: The high level has transactions, each of which is a sequence of operations accessing data items. At the low level, these transactions are translated into executions in which a sequence of events apply primitive operations (or primitives) to base objects, containing the data and the meta-data needed for the implementation.

STM Properties. The interval of a transaction $T$ is the execution interval that starts at the first event of $T$ and ends at the last event of $T$, if there is one, taken by the process executing the algorithm for $T$. If $T$ does not have a last event in the execution, then the interval of $T$ is the (possibly infinite) execution interval starting at the first event of T. Two transactions overlap if their intervals overlap. A configuration $C$ is quiescent if no transaction is pending in $C$, i.e., it is not inside the interval of any transaction.

An STM is serializable if transactions appear to execute sequentially, one after the other [21]; we assume that the serialization preserves the per-process order, i.e., transactions of the same process maintain their order. An STM is strictly serializable if this order preserves the order of non-overlapping transactions [21]; this notion is called orderpreserving serializability in [25], and is the analogue of linearizability [15] for transactions. Note that strict serializability is implied by the opacity correctness condition, recently defined for transactional memory [10].

We assume that a transaction terminates successfully if it runs alone from a quiescent configuration. This property is satisfied by obstruction-free STM implementations, in which a process that eventually runs alone for long enough makes progress, i.e., transactions terminate successfully when eventually executing solo [13]. This property is also satisfied by STM implementations that are weakly progressive [11], in which a transaction that does not encounter conflicts has to terminate successfully; note that blocking STM implementations like TL2 [6] are weakly progressive.

Memory disjoint-access parallelism. An important property STM implementations have to provide is allowing unrelated transactions to progress independently, even if they are concurrent. Below, we formally define what it means for two transactions to be unrelated through a conflict graph that represents the relations between transactions. Then we define disjoint-access parallelism, a property that captures the intuition that an implementation should not cause two transactions, which are unrelated at the high-level, to simultaneously access the same low-level shared memory.

The conflict graph of an execution interval $I$ is an undirected graph in which vertices represent data items and edges connect data items if they are accessed by the same transaction. If $I$ overlaps the execution interval of a transaction $T$, and the data items $\imath_{1}$ and $\imath_{2}$ are in the data set of $T$, the graph includes an edge between the vertices representing $\imath_{1}$ and $\imath_{2}$.

Two transactions $T_{1}$ and $T_{2}$ are disjoint-access if there is no path between an item in the data set of $T_{1}$ and an item in the data set of $T_{2}$, in the conflict graph of the minimal execution interval containing the intervals of $T_{1}$ and $T_{2}$.

Two events contend on a base object $o$ if they both access $o$, and at least one of them applies a non-trivial primitive to $o$. Two processes concurrently contend on a base object $o$ if they have pending events at the same configuration that contend on $o$.

DeFinition 1. An STM implementation is weakly disjointaccess parallel if two processes $p_{1}$ and $p_{2}$, executing transactions $T_{1}$ and $T_{2}$, concurrently contend on the same base object, only if $T_{1}$ and $T_{2}$ are not disjoint-access.

This definition captures the first condition of the disjointaccess parallelism property of Israeli and Rappoport [17], in accordance with most of the literature (cf. [14]). Our definition is weaker than their definition, as it allows two processes to apply a trivial primitive on the same base object, e.g., read, when executing two transactions even if they are disjoint-access. Moreover, our definition only prohibits concurrent contending accesses, allowing transactions to contend on a base object $o$ at different points of the execution; we shall see in Lemma 2 that, under some conditions, these transactions can be made to concurrently contend on $o$.

The original definition [17] also restricts the impact of concurrent transactions on the step complexity of a transaction; our results do not rely on this additional condition, making them stronger.

\section{STRICTLY SERIALIZABLE STMS}

\subsection{Impossibility of Invisible Read-Only Trans- actions}

A read-only transaction is invisible if its algorithm only applies trivial primitives to base objects. We prove that in a disjoint-access parallel STM implementation with invisible read-only transactions, some read-only transaction will not terminate successfully in a finite number of steps; this is formally stated in Theorem 4 .

Specifically, we construct an infinite execution of a readonly transaction. This execution consists of a single readonly transaction with one complete update transaction between any pair of consecutive steps by the read-only transaction; an update is a transaction with a singleton write set and an empty read set. We first define a special (finite) execution of this form, called flippable, and show that such a read-only transaction cannot terminate successfully. Then we show how a flippable execution can be repeatedly extended to construct successively longer flippable executions.

An execution is called flippable since there are two similar executions in which we flip the position of two update transactions and one of the executions is indistinguishable from the original execution. One type of flipped execution is called a forward flip since it moves an update transaction forward in the execution, while other is called a backward flip since it defers the execution of an update transaction. Formally:

Definition 2. A flippable execution of length $k$ with $t$ updaters is a finite execution $E_{k}=U_{0} s_{1} U_{1} \ldots s_{k} U_{k}$ executed 


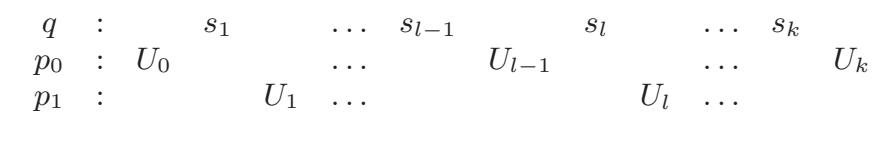

$$
\begin{aligned}
& \text { (a) } E_{k} \text {. }
\end{aligned}
$$

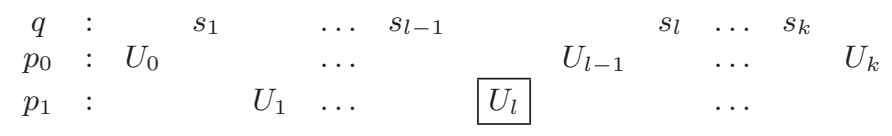

(b) Forward flip: $U_{l}$ is performed before $U_{l-1} s_{l}$.

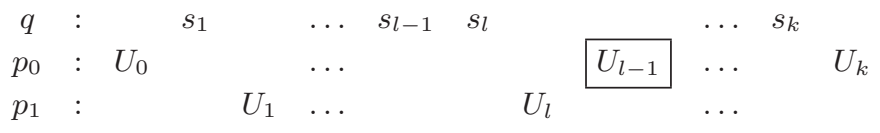

(c) Backward flip: $U_{l-1}$ is performed after $s_{l} U_{l}$.

Figure 2: A flippable execution of length $k$ with two updaters: Figure 2(a) shows a flippable execution $E_{k}$; Figure 2(b) shows the forward flip execution of $E_{k}$, where the update transaction $U_{l}$ by process $p_{1}$ is executed before the update transaction $U_{l-1}$ by process $p_{0}$ and before the step $s_{l}$ of the read-only transaction; Figure 2(c) shows the backward flip execution of $E_{k}$, where the update $U_{l-1}$ by process $p_{0}$ is deferred after the update transaction $U_{l}$ by process $p_{1}$ and after the step $s_{l}$ of the read-only transaction.

by processes $p_{0}, \ldots, p_{t-1}$ executing update transactions and process $q$ executing a read-only transaction, which reads and returns the value of $t$ data items $\imath_{0} \ldots \imath_{t-1}$. The execution $E_{k}$ satisfies all the following conditions:

1. for $j=1, \ldots, k, s_{j}$ is a single step by $q$,

2. for $j=0, \ldots, k, U_{j}$ is a solo execution of a complete update transaction, in which process $p_{h} \in\left\{p_{0}, \ldots, p_{t-1}\right\}$, writes $j+1$ to the data item $\imath_{h}$

3. consecutive updates are executed by different processes, and

4. for any $l, 0<l \leq k$, the execution

$$
E_{k}=U_{0} s_{1} U_{1} \ldots s_{l-1} U_{l-1} s_{l} U_{l} \ldots s_{k} U_{k}
$$

is indistinguishable to all processes from one of the following executions:

$$
\overleftarrow{F}_{l}=U_{0} s_{1} U_{1} \ldots s_{l-1} U_{l} U_{l-1} s_{l} \ldots s_{k} U_{k}
$$

in which the update transaction $U_{l}$ is executed before $U_{l-1} s_{l}$ instead of after $U_{l-1} s_{l}$ (forward flip) or

$$
\vec{F}_{l}=U_{0} s_{1} U_{1} \ldots s_{l-1} s_{l} U_{l} U_{l-1} \ldots s_{k} U_{k}
$$

in which the update transaction $U_{l-1}$ is executed after $s_{l} U_{l}$ instead of before $s_{l} U_{l}$ (backward flip).

Figures 2(b) and 2(c) present the forward and the backward flips of the execution in Figure 2(a).

This definition, and the structure of our proof, is similar to the lower bound of Attiya, Ellen and Fatourou [2] on the step complexity of update operations in implementations of atomic snapshot objects. The main difference is that our definition of a flippable execution has two types of flipped executions, and $t$ processes executing update transactions instead of just two.

The next lemma is proved by arguments similar to those applied in [2], extended to handle the possibility of two kinds of flips (forward and backward).
LEMma 1. The read-only transaction in a flippable execution does not terminate successfully.

Proof. Let $E_{k}=U_{0} s_{1} U_{1} \ldots s_{k} U_{k}$ be a flippable execution. Assume, towards a contradiction, that $q$ successfully terminates its read-only transaction in $E_{k}$, with a result $\vec{v}=\left(v_{0}, \ldots, v_{t-1}\right)$. Since the update transactions in the execution $E_{k}$ do not overlap, they must be serialized in the order $U_{0}, \ldots, U_{l}$. Since all steps of the read-only transaction by $q$ are after $U_{0}$ and before $U_{k}$, it has a unique serialization point between $U_{l-1}$ and $U_{l}$, for some $l, 1 \leq l \leq k$. Let $\imath_{h}$ be the item written by $U_{l-1}$, and recall that $U_{l-1}$ writes $l$ to $\imath_{h}$; hence $v_{h}=l$.

The execution $E_{k}$ is indistinguishable to process $q$ from $F_{l}$, which is either the forward flip

$$
\overleftarrow{F}_{l}=U_{0} s_{1} U_{1} \ldots s_{l-1} U_{l} U_{l-1} s_{l} s_{l+1} \ldots U_{k}
$$

in which update $U_{l}$ is executed before $U_{l-1} s_{l}$ instead of after $U_{l-1} s_{l}$; or the backward flip

$$
\vec{F}_{l}=U_{0} s_{1} U_{1} \ldots s_{l-1} s_{l} U_{l} U_{l-1} s_{l+1} \ldots U_{k}
$$

in which update $U_{l-1}$ is executed after $s_{l} U_{l}$ instead of before $s_{l} U_{l}$. Hence, the read-only transaction executed by $q$ in $F_{l}$ returns the same result, $\vec{v}$, as in $E_{k}$.

Since the update transactions do not overlap in $F_{l}$, they are serialized in the order $U_{0}, \ldots, U_{l}, U_{l-1}, \ldots, U_{k}$, that is, the same as for $E_{k}$, except that $U_{l-1}$ and $U_{l}$ are flipped. Since two consecutive update transactions are on different items, the values of $\left\{\imath_{0}, \ldots, \imath_{t-1}\right\}$ are the same after both update transactions have been executed, no matter which has been executed first. Hence, at all points in the serialization of $F_{l}$, except between $U_{l}$ and $U_{l-1}$, the value of all items $\left\{\imath_{0}, \ldots, \imath_{t-1}\right\}$ is the same as its value in the corresponding points in the serialization of $E_{k}$. Thus, the read-only transaction of $q$ can only serialized after $U_{l}$ and before $U_{l-1}$ in $F_{l}$. However, since $U_{l-1}$ is the first write of $l$ to $\imath_{h}$, the value of $\imath_{h}$ is not $l$ before $U_{l-1}$, and hence, the read-only transaction executed by $q$ cannot be serialized between $U_{l}$ and 

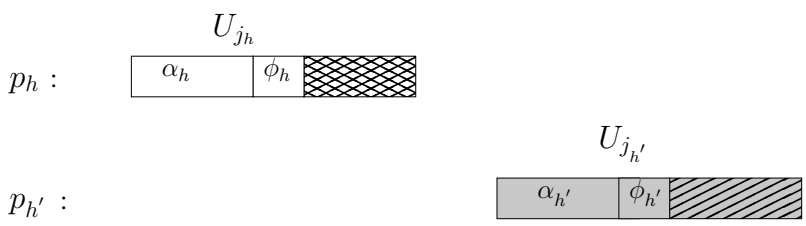

(a) Serial execution of $U_{j_{h}}$ and $U_{j_{h^{\prime}}}$ from configuration $C$

$p_{h}$

$\alpha_{h}$

$p_{h^{\prime}}:$

\begin{tabular}{l|l}
$\alpha_{h^{\prime}}$ & $\phi_{h^{\prime}}$
\end{tabular}

(b) Overlapping execution of $U_{j_{h}}$ and $U_{j_{h^{\prime}}}$ from configuration $C$

\section{Figure 3: Illustration for the proof of Lemma 2}

$U_{l-1}$. This contradicts the assumption that the read-only transaction terminates successfully.

Lemma 3 (below) proves that when read-only transactions are invisible, we can inductively construct a flippable execution. The crux of this lemma is quite different from [2], as it relies on weakly disjoint-access parallelism. A critical step in the proof is provided by the next lemma, showing that in a weakly disjoint-access parallel STM, two consecutive updates by different processes on different items cannot contend on the same base objects. The proof of the lemma shows that two such consecutive updates can be perturbed to concurrently contend on the same base object.

LEMma 2. Given a weakly disjoint-access parallel STM implementation and a quiescent configuration $C$, consider the consecutive execution of two update transactions $U_{j_{h}} U_{j_{h^{\prime}}}$, executed by a process $p_{h}$ on an item $\imath_{h}$ and by process $p_{h^{\prime}}$ on an item $\imath_{h^{\prime}}, h \neq h^{\prime}$, respectively, from $C$. Then $p_{h}$ and $p_{h^{\prime}}$ do not contend on the same base object when executing $U_{j_{h}}$ and $U_{j_{h^{\prime}}}$.

Proof. Assume, towards a contradiction, that $p_{h}$ and $p_{h^{\prime}}$ contend on a base object when executing $U_{j_{h}} U_{j_{h^{\prime}}}$ from a quiescent configuration $C$. If in $U_{j_{h}}, p_{h}$ applies a nontrivial primitive to a base object on which they contend, let $\phi_{h}$ be the last event in $U_{j_{h}}$ in which $p_{h}$ applies such a primitive, say, to base object $o$. Let $\phi_{h^{\prime}}$ be the first event in $U_{j_{h^{\prime}}}$ that accesses $o$. Otherwise, $p_{h}$ only applies trivial primitives in $U_{j_{h}}$ to base objects on which it contends with $p_{h^{\prime}}$ in $U_{j_{h^{\prime}}}$; let $\phi_{h^{\prime}}$ be the first event in $U_{j_{h^{\prime}}}$ in which $p_{h^{\prime}}$ applies a non-trivial primitive to some base object, say, $o$, on which they contend. Let $\phi_{h}$ be the last event of $p_{h}$ in $U_{j_{h}}$ that accesses $o$. In both cases, denote by $\alpha_{h} \phi_{h}$ the prefix of the execution of $U_{h}$ from $C$ and by $\alpha_{h^{\prime}} \phi_{h^{\prime}}$ the prefix of the execution of $U_{h^{\prime}}$ after $U_{h}$ (see Figure 3(a)).

We now consider an overlapping execution of the update transactions $U_{j_{h}}$ and $U_{j_{h^{\prime}}}$, by processes $p_{h}$ and $p_{h^{\prime}}$, from $C$. We argue that $p_{h}$ and $p_{h^{\prime}}$ perform the same steps up to the events $\phi_{h}$ and $\phi_{h^{\prime}}$, and as shown in Figure $3(\mathrm{~b}), p_{h}$ and $p_{h^{\prime}}$ concurrently contend on base object $o$.

In more detail, consider the execution $\alpha_{h} \alpha_{h^{\prime}}$ from $C$, in which $p_{h}$ executes $U_{j_{h}}$ until it is about to perform $\phi_{h}$, and then $p_{h^{\prime}}$ executes $U_{h_{h^{\prime}}}$ until it is about to perform $\phi_{h^{\prime}}$. Clearly, $p_{h}$ is about to perform $\phi_{h}$ also after $\alpha_{h} \alpha_{h^{\prime}}$. By construction, the execution interval $\alpha_{h} \alpha_{h^{\prime}}$ from $C$ is indistinguishable to $p_{h^{\prime}}$ from the execution interval $U_{j_{h}} \alpha_{h^{\prime}}$ from $C$. Hence, $p_{h^{\prime}}$ is about to perform the event $\phi_{h^{\prime}}$ also after $\alpha_{h} \alpha_{h^{\prime}}$, that is, $p_{h^{\prime}}$ and $p_{h}$ concurrently contend on $o$. However, the conflict graph of the execution interval $\alpha_{h} \alpha_{h^{\prime}} \phi_{h^{\prime}} \phi_{h}$ does not contain a path between the data sets of $U_{j_{h}}$ and $U_{j_{h^{\prime}}}$, contradicting the assumption that the implementation is weakly disjoint-access parallel.

Since two consecutive updates do not contend on the same base object, we can construct an execution where either the previous update is deferred or the next update is moved forward in the execution without affecting the single step of the read-only transaction in between them. This allows us to inductively construct a flippable execution, in the proof of the next lemma.

Lemma 3. For every $k \geq 0$, every weakly disjoint-access parallel implementation of an STM with invisible read-only transactions, has a flippable execution $E_{k}=U_{0} s_{1} U_{1} s_{2} \ldots U_{k}$ with two updaters $p_{0}$ and $p_{1}$, which is indistinguishable to $p_{0}$ and $p_{1}$ from the execution $E_{k}^{\prime}=U_{0} U_{1} \ldots U_{k}$ in which only $p_{0}$ and $p_{1}$ take steps.

Proof. The proof is by induction on the length $k$ of the flippable execution $E_{k}$ executed by a process $q$ and two updaters $p_{0}$ and $p_{1}$ on two items $\left\{\imath_{0}, \imath_{1}\right\}$. In the base case, $k=0$, the lemma holds with a solo execution of $U_{0}$, an update transaction by $p_{0}$ that writes 1 to $\imath_{0}$. $U_{0}$ successfully terminates since it runs solo from a quiescent configuration.

For the induction step, consider a flippable execution of length $k, E_{k}=U_{0} s_{1} U_{1} s_{2} \ldots U_{k}$. By Lemma 1 , the read-only transaction does not terminate successfully in $E_{k}$. Let $s_{k+1}$ be the next step by $q$. Assume $U_{k}$ is executed by $p_{h^{\prime}}$ and let $h=1-h^{\prime}$; note that $h \neq h^{\prime}$. Let $E_{k+1}=E_{k} s_{k+1} U_{k+1}$, where process $p_{h}$ writes $k+2$ to $\imath_{h}$ in the update transaction $U_{k+1}$. Note that $U_{k+1}$ terminates successfully, by our progress condition; although the configuration at the end of $E_{k+1}=E_{k} s_{k+1}$ is not quiescent, it is indistinguishable from the quiescent configuration at the end of $E_{k}^{\prime}$.

Since the read-only transaction by $q$ is invisible, $E_{k+1}$ is indistinguishable to $p_{0}$ and $p_{1}$ from the execution $E_{k}^{\prime} U_{k+1}$.

It remains to prove that for every $l, 0<l \leq k+1$, the execution $E_{k+1}$ is indistinguishable to all processes from either $\overleftarrow{F}_{l}$ or $\vec{F}_{l}$. For every $l, 0<l \leq k$, by the inductive assumption, the execution

$$
E_{k}=U_{0} s_{1} U_{1} \ldots s_{l-1} U_{l-1} s_{l} U_{l} \ldots s_{k} U_{k}
$$

is indistinguishable to all processes from the flipped execution $F_{l}$ which is either

$$
\overleftarrow{F}_{l}=U_{0} s_{1} U_{1} \ldots s_{l-1} U_{l} U_{l-1} s_{l} \ldots U_{k}
$$

or

$$
\vec{F}_{l}=U_{0} s_{1} U_{1} \ldots s_{l-1} s_{l} U_{l} U_{l-1} \ldots s_{k} U_{k} .
$$

In particular, the configurations at the end of the two executions $E_{k}$ and $F_{l}$ are the same. Hence, $E_{k+1}=E_{k} s_{k+1} U_{k+1}$ and $F_{l} s_{k+1} U_{k+1}$ are indistinguishable to all processes.

To prove the condition for $l=k+1$, let $C_{k-1}^{\prime}$ be the configuration at the end of $E_{k-1}^{\prime} ; C_{k-1}^{\prime}$ is quiescent, and Lemma 2 implies that $p_{h^{\prime}}$ and $p_{h}$ do not contend on the same base object when executing $U_{k}$ followed by $U_{k+1}$ from $C_{k-1}^{\prime}$, namely, in the suffix of $E_{k+1}^{\prime}$. By the indistinguishability of $E_{k+1}^{\prime}$ and $E_{k+1}, p_{h^{\prime}}$ and $p_{h}$ do not contend on the same base 
object while executing $U_{k}$ and $U_{k+1}$ also in the execution $E_{k+1}$. Moreover, if $q$ accesses a base object $o$ in $s_{k+1}$, then either at least one of the two processes $p_{h}$ or $p_{h^{\prime}}$ does not access $o$ in $U_{k+1}$ or $U_{k}$, respectively, or they both apply a trivial primitive to $o$. In the former case, if $p_{h}$ does not access $o$ in $U_{k+1}$ then

$$
\overleftarrow{F}_{k+1}=U_{0} s_{1} U_{1} \ldots s_{k} U_{k+1} U_{k} s_{k+1}
$$

is indistinguishable to all processes from $E_{k+1}$, while if $p_{h^{\prime}}$ does not access $o$ in $U_{k}$, then

$$
\vec{F}_{k+1}=U_{0} s_{1} U_{1} \ldots s_{k} s_{k+1} U_{k+1} U_{k}
$$

is indistinguishable to all processes from $E_{k+1}$. If both $p_{h}$ and $p_{h^{\prime}}$ apply a trivial primitive to $o$, then both flipped executions, $\overleftarrow{F}_{k+1}$ and $\vec{F}_{k+1}$, are indistinguishable to all processes from $E_{k+1}$.

The impossibility result follows from Lemmas 1 and 3 .

THEOREM 4. There is no weakly disjoint-access parallel implementation with invisible read-only transactions of a strictly serializable STM, in which read-only transactions always terminate successfully.

The impossibility result stated in Theorem 4 holds also for opaque STMs [10], since opacity implies strict serializability.

\subsection{Lower Bound for Read-Only Transactions}

The technique of the previous section can be extended to prove that a read-only transaction of $t$ items in a disjointaccess parallel STM implementation, which successfully terminates in a finite number of steps, must apply non-trivial primitives to $t-1$ base objects; this assumes that there are at least $t+1$ processes.

The proof of Lemma 1 - showing that the read-only transaction in a flippable execution cannot terminate successfullydoes not rely on the fact that the read-only transaction is invisible, and the lemma continues to hold. On the other hand, we must modify the proof showing the existence of the flippable execution.

This result relies on a stronger notion of disjoint-access parallelism, which requires two transactions to be connected (in the conflict graph) even if they both just apply a trivial primitive to the same base object. (This is the definition in [17].) Two processes concurrently access a base object $o$ if both have a pending access to $o$ at some configuration.

Definition 3. An STM implementation is disjoint-access parallel if two processes $p_{1}, p_{2}$ concurrently access the same base object when executing transactions $T_{1}$ and $T_{2}$, respectively, only if $T_{1}$ and $T_{2}$ are not disjoint-access.

Since we now put a stronger requirement on disjoint-access parallel STM implementations, Lemma 2, assuming a weaker requirement, still holds.

We first show (in Lemma 5) that, in a disjoint-access parallel STM implementation, two update transactions executed by different processes on different items do not access a common base object when each of them runs solo from a quiescent configuration. This is used in Lemma 6 to prove the existence of a flippable execution, when a read-only transaction of $t$ data items applies non-trivial primitives to at most $t-2$ base objects.

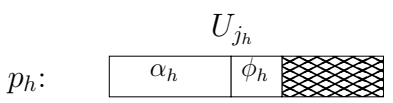

(a) Solo execution of $U_{j_{h}}$ from configuration $C$

\begin{tabular}{c}
\multicolumn{1}{c}{$U_{j_{h^{\prime}}}$} \\
$p_{h^{\prime}}: \quad \alpha_{h^{\prime}}$ \\
\hline$\alpha_{h^{\prime}}$
\end{tabular}

(b) Solo execution of $U_{j_{h^{\prime}}}$ from configuration $C$

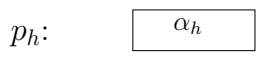
$p_{h^{\prime}}$

\begin{tabular}{l|l|}
$\alpha_{h^{\prime}}$ & $\phi_{h^{\prime}}$ \\
\hline
\end{tabular}

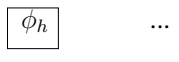

(c) Overlapping execution of $U_{j_{h}}$ and $U_{j_{h^{\prime}}}$ from configuration $C$

Figure 4: Illustration for the proof of Lemma 5.

LEMMA 5. Given a disjoint-access parallel STM implementation and a quiescent configuration $C$, consider the execution of an update transaction $U_{j_{h}}$ to the item $\imath_{h}$ by process $p_{h}$, and an update transaction $U_{j_{h^{\prime}}}$ to the item $\imath_{h^{\prime}}$ by process $p_{h^{\prime}}, h \neq h^{\prime}$, from $C$. Then, $p_{h}$ and $p_{h^{\prime}}$ do not access a common base object when executing $U_{j_{h}}$ and $U_{j_{h^{\prime}}}$, respectively.

Proof. Assume, towards a contradiction, that $p_{h}$ and $p_{h^{\prime}}$ access the same base object while executing $U_{j_{h}}$ and $U_{j_{h^{\prime}}}$, respectively, from $C$. Let $o$ be the first base object accessed by $p_{h}$ that is also accessed by $p_{h^{\prime}}$. Let $\alpha_{h} \phi_{h}$ be the prefix of the execution of $U_{j_{h}}$ from $C$, where $\phi_{h}$ is the first event in which $p_{h}$ accesses $o$ (see Figure $4(\mathrm{a})$ ). Let $\alpha_{h^{\prime}} \phi_{h^{\prime}}$ be the prefix of the execution of $U_{j_{h^{\prime}}}$ from $C$, where $\phi_{h^{\prime}}$ is the first access of $p_{h^{\prime}}$ to $o$ (see Figure 4(b)).

Consider the execution $\alpha_{h} \alpha_{h^{\prime}}$ from $C$, where $p_{h}$ executes $U_{j_{h}}$ until it is about to access $o$, and then $p_{h^{\prime}}$ executes $U_{j_{h^{\prime}}}$ until it is about to access $o$ (see Figure 4(c)). By construction, the execution $\alpha_{h} \alpha_{h^{\prime}}$ from $C$ is indistinguishable to $p_{h}$ and $p_{h^{\prime}}$ from the corresponding executions $\alpha_{h}$ and $\alpha_{h^{\prime}}$ from $C$. Thus, $p_{h^{\prime}}$ has the event $\phi_{h^{\prime}}$ pending and $p_{h}$ has the event $\phi_{h}$ pending after $\alpha_{h} \alpha_{h^{\prime}}$; thus, $p_{h^{\prime}}$ and $p_{h}$ have concurrently pending accesses to $o$. However, in the conflict graph of the execution interval $\alpha_{h} \alpha_{h^{\prime}} \phi_{h^{\prime}} \phi_{h}$ from $C$, there is no path between the data sets of $U_{j_{h}}$ and $U_{j_{h^{\prime}}}$, contradicting the assumption that the implementation is disjoint-access parallel.

We show that at any point during the execution of the read-only transaction, there is a process that can write to its item without accessing any base object to which $q$ applies non-trivial primitives, thus making the read-only transaction "invisible" to the other processes. Note that, by the definition of a flippable execution, each process always updates the same item. We prove such a process exists by applying a "pigeon hole" argument to show that the process does not access any base object to which the read-only transaction applies non-trivial primitives. Since there are $t-1$ processes to choose from, each accessing a different item, and since the read-only transaction applies non-trivial primitives to at most $t-2$ base objects, at least two update transactions by different processes access the same base object, which can be shown to violate disjoint-access parallelism. 
Lemma 6. For every $k \geq 0$, a disjoint-access parallel implementation of an STM in which a read-only transaction of $t$ data items applies non-trivial primitives to at most $t-2$ base objects, has a flippable execution $E_{k}=U_{0} s_{1} U_{1} s_{2} \ldots U_{k}$ with $t$ updaters, which is indistinguishable to $p_{0}, \ldots, p_{t-1}$ from the execution $E_{k}^{\prime}=U_{0} U_{1} \ldots U_{k}$ in which only $p_{0}, \ldots, p_{t-1}$ take steps.

Proof. The proof is by induction on the length $k$ of the flippable execution $E_{k}$. The base case is when $k=0$. The lemma holds with a solo execution of an update transaction, $U_{0}$, by process $p_{0}$ that writes 1 to $\imath_{1}$. $U_{0}$ successfully terminates since it runs solo from a quiescent configuration.

For the induction step, consider a flippable execution of length $k, E_{k}=U_{0} s_{1} U_{1} s_{2} \ldots U_{k}$, which is indistinguishable to $p_{0}, \ldots, p_{t-1}$ from the execution $E_{k}^{\prime}=U_{0} U_{1} \ldots U_{k}$. By Lemma 1 , the read-only transaction does not terminate successfully in $E_{k}$. Let $s_{k+1}$ be the next step by $q$ and let $C_{k+1}$ denote the configuration at the end of $E_{k} s_{k+1}$; also, let $C_{k+1}^{\prime}$ be the configuration at the end of $E_{k}^{\prime}$.

The process $p_{h}$ to execute $U_{k+1}$ is chosen from $p_{0}, \ldots, p_{t-1}$ such that $p_{h}$ did not execute $U_{k}$ and a solo execution of $U_{k+1}$ from $C_{k+1}$ by $p_{h}$ does not access any base objects to which $q$ applies non-trivial primitives in $E_{k} s_{k+1}$. Note that this transaction must terminate successfully, by our progress condition; although $C_{k+1}$ is not quiescent, it is indistinguishable from $C_{k+1}^{\prime}$, which is quiescent.

We claim such a process exists. Assume, towards a contradiction, that for every process $p_{h_{k+1}}, h_{k+1} \neq h_{k}$, the solo execution by $p_{h_{k+1}}$ from $C_{k+1}$ of the update transaction that writes $k+2$ to $\imath_{h_{k+1}}$ accesses a base object to which $q$ applies a non-trivial primitive in $E_{k} s_{k+1}$. We consider $t-1$ possible processes, each writing to a different item. Since the readonly transaction applies non-trivial primitives to at most $t-2$ base objects, at least two update transactions executed by different processes $p_{h}$ and $p_{h^{\prime}}$ to different items $\imath_{h}$ and $\imath_{h^{\prime}}$, starting from configuration $C_{k+1}$, access the same base object in their first access to a base object to which $q$ applies a non-trivial primitive. Recall that $C_{k+1}^{\prime}$ is quiescent. Since the execution $E_{k} s_{k+1}$ is indistinguishable to processes $p_{h}$ and $p_{h^{\prime}}$ from the execution $E_{k}^{\prime}$, they access the same base object also when executing the update transactions from $C_{k+1}^{\prime}$, which by Lemma 5 , violates the assumption that the implementation is disjoint-access parallel.

Pick some process $p_{h_{k+1}}, h_{k+1} \neq h_{k}$, that does not access any base objects to which $q$ applies non-trivial primitives in $E_{k} s_{k+1}$; let $U_{k+1}$ be an update by $p_{h_{k+1}}$ that writes $k+2$ to $\imath_{h_{k+1}}$ and denote $E_{k+1}=E_{k} s_{k+1} U_{k+1}$.

Next, we prove that the execution $E_{k+1}$ is indistinguishable to $p_{0}, \ldots, p_{t-1}$ from the execution $E_{k+1}^{\prime}$. This holds for processes other than $p_{h_{k+1}}$ by the inductive assumption and since these processes take no steps in the suffix of this execution. For $p_{h_{k+1}}$, this holds by the inductive assumption and since the solo execution $U_{k+1}$ of an update transaction by $p_{h_{k+1}}$ does not access base objects to which $q$ applies a non-trivial primitive in $E_{k} s_{k+1}$.

It remains to prove that for every $l, 0<l \leq k+1$, the execution $E_{k+1}$ is indistinguishable to all processes from the flipped execution $F_{l}$ which is either $\overleftarrow{F}_{l}$ or $\vec{F}_{l}$, as defined in Definition 2. For every $l, 0<l \leq k$, by the inductive assumption, the execution

$$
E_{k}=U_{0} s_{1} U_{1} \ldots s_{l-1} U_{l-1} s_{l} U_{l} \ldots s_{k} U_{k}
$$

is indistinguishable to all processes from the flipped execu- tion $F_{l}$ which is either

$$
\overleftarrow{F}_{l}=U_{0} s_{1} U_{1} \ldots s_{l-1} U_{l} U_{l-1} s_{l} \ldots U_{k}
$$

or

$$
\vec{F}_{l}=U_{0} s_{1} U_{1} \ldots s_{l-1} s_{l} U_{l} U_{l-1} \ldots s_{k} U_{k} .
$$

In particular, the configurations at the end of the two executions $E_{k}$ and $F_{l}$ are the same. Hence, the executions $E_{k+1}=E_{k} s_{k+1} U_{k+1}$ and $F_{l} s_{k+1} U_{k+1}$ are indistinguishable to all processes.

For $l=k+1$, consider the flipped executions $\overleftarrow{F}_{k+1}$ and $\vec{F}_{k+1}$. The configuration $C_{k-1}^{\prime}$ at the end of $E_{k-1}^{\prime}$ is quiescent. Any STM implementation which is disjoint-access parallel is also weakly disjoint-access parallel, hence we can apply Lemma 2 to deduce that $p_{h_{k}}$ and $p_{h_{k+1}}$ do not contend on, and hence do not access the same base object while executing $U_{k}$ and $U_{k+1}$ from $C_{k-1}^{\prime}$. The indistinguishability property implies that $p_{h_{k}}$ and $p_{h_{k+1}}$ do not access the same base object while executing $U_{k}$ and $U_{k+1}$ also in $E_{k+1}$.

Moreover, if $q$ applies a trivial primitive to some base object $o$ in $s_{k+1}$, then either at least one of the two processes $p_{h_{k+1}}$ and $p_{h_{k}}$ does not access $o$ in $U_{k+1}$ and in $U_{k}$ respectively, or they both apply a trivial primitive to $o$. In the former case, if $p_{h_{k+1}}$ does not access in $U_{k+1}$ any object that $q$ accesses in $s_{k+1}$, then

$$
\overleftarrow{E}_{k+1}=U_{0} s_{1} U_{1} \ldots s_{k} U_{k+1} U_{k} s_{k+1}
$$

is indistinguishable to all processes from $E_{k+1}$, while if $p_{h_{k}}$ does not access in $U_{k}$ any object that $q$ accesses in $s_{k+1}$, then

$$
\vec{E}_{k+1}=U_{0} s_{1} U_{1} \ldots s_{k} s_{k+1} U_{k+1} U_{k}
$$

is indistinguishable to all processes from $E_{k+1}$. If $p_{h_{k+1}}$ and $p_{h_{k}}$ apply a trivial primitive to $o$, then both flipped executions are indistinguishable to all processes from $E_{k+1}$.

The lower bound follows:

THEOREM 7. In a strict serializable disjoint-access parallel STM implementation for $t+1$ processes, where all readonly transactions terminate successfully, some read-only transaction of $t \geq 2$ data items applies non-trivial primitives to at least $t-1$ base objects.

This lower bound holds also for opaque STMs, since opacity implies strict serializability.

\section{EXTENDING THE RESULTS TO WEAKER CONSISTENCY CONDITIONS}

In this section, we show that both Theorem 4 and Theorem 7 hold for weaker consistency conditions, namely, serializability and snapshot isolation. This uses an additional process.

Recall that an STM is serializable if transactions appear to execute sequentially, one after the other; we further require that transactions of the same process preserve their order (per-process order).

Given a flippable execution $E_{k}=U_{0} s_{1} U_{1} \ldots s_{k} U_{k}$, we construct an augmented flippable execution

$$
\widehat{E}_{k}=U_{0} s_{1} S_{1}^{*} U_{1} \ldots s_{k} S_{k}^{*} U_{k},
$$

where an additional process $q^{\prime}$ performs invisible read-only transactions. For every $j \in\{1, \ldots, k\}, q^{\prime}$ performs solo a 


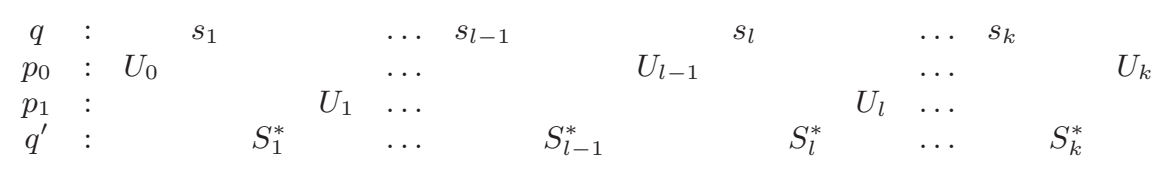

Figure 5: An augmented flippable execution $\widehat{E}_{k}$ derived from the flippable execution $E_{k}$ of Figure 2.

sequence $S_{j}^{*}$ of read-only transactions after the event $s_{j}$ by process $q$ and before the update $U_{j}$. Each read-only transaction in $S_{j}^{*}$ accesses the items $\imath_{f_{j-1}}$ and $\imath_{f_{j}}$ updated by $U_{j-1}$ and $U_{j}$. The result of the last read-only transaction in the sequence $S_{j}^{*}$, denoted $S_{j}$, is the value written by $U_{j-1}$ to $\imath_{f_{j-1}}$ and the last value of $\imath_{f_{j}}$ before $U_{j}$ updates it.

Figure 5 shows the augmented flippable execution obtained by augmenting the flippable execution $E_{k}$ of Figure 2 with sequences of read-only transactions performed by an additional process $q^{\prime}$.

We apply the per-process ordering of transactions to prove that the read-only transactions of $q^{\prime}$ must eventually read the latest value written in $U_{j-1}$, and thus, $S_{j}^{*}$ is finite.

LEMMA 8. Consider an augmented flippable execution of length $k \geq 0, \widehat{E}_{k}=U_{0} s_{1} S_{1}^{*} U_{1} \ldots s_{k} S_{k}^{*} U_{k}$. In any serialization of $\widehat{E}_{k}$ that preserves the per-process order, $U_{0}, U_{1}, \ldots$, $U_{k}$ appear in their order of execution.

Proof. We show, by induction on $\ell$, that $U_{0}, U_{1}, \ldots, U_{\ell}$ appear in their order of execution. The base case is trivial.

For the induction step, consider $U_{\ell+1}$. By the induction assumption, the updates $U_{0}, U_{1}, \ldots, U_{\ell}$ are serialized by their execution order in $\widehat{E}_{k}$. By construction $S_{\ell+1}^{*}$ accesses the items $\imath_{f_{\ell}}$ and $\imath_{f_{\ell+1}}$ repeatedly up to a read-only transaction $S_{\ell+1}$, which returns the value written by $U_{\ell}$ and the last value of $\imath_{f_{\ell+1}}$ before the one written by $U_{\ell+1}$.

$S_{\ell+1}^{*}$ is finite since the STM is serializable and so, eventually, some transaction must return the latest values written to $\imath_{f_{\ell}}$ and $\imath_{f_{\ell+1}}$, and by the induction assumption, $U_{\ell}$ is the last to write to $\imath_{f_{\ell}}$. Moreover, $S_{\ell+1}$ completes before $U_{\ell+1}$ starts, so it cannot return the value written by $U_{\ell+1}$, since due to serializability, a read operation can not return a value not written.

Since each data item is written by a different process, and due to per-process order, $U_{\ell+1}$ can not be serialized before the last update of $\imath_{f_{\ell+1}}$ preceding $U_{\ell+1}$.

Moreover, $U_{\ell+1}$ can not be serialized after this update and before $S_{\ell+1}$, since $S_{\ell+1}$ does not return the value written by $U_{\ell+1}$. Hence, $U_{\ell+1}$ is serialized after $S_{\ell+1}$.

We use Lemma 8 to prove the analogue of Lemma 1.

LEMMA 9. Consider an augmented fippable execution of length $k \geq 0$ with $t$ updaters, $\widehat{E}_{k}=U_{0} s_{1} S_{1}^{*} U_{1} \ldots s_{k} S_{k}^{*} U_{k}$. If the read-only transactions by process $q^{\prime}$ are invisible, then the read-only transaction by process $q$ does not terminate successfully.

Proof. Assume, towards a contradiction, that the readonly transaction of process $q$ in $\widehat{E}_{k}$ terminates successfully and returns a value $\vec{v}=\left(v_{0}, \ldots, v_{t-1}\right)$, which does not violate serializability. Let the augmented flippable execution $\widehat{E}_{k}=U_{0} s_{1} S_{1}^{*} U_{1} \ldots s_{k} S_{k}^{*} U_{k}$ correspond to a flippable execution $E_{k}=U_{0} s_{1} U_{1} \ldots s_{k} U_{k}$.
By Lemma 8, the updates in $\widehat{E}_{k}$ are serialized in the order $U_{0}, U_{1}, \ldots, U_{k}$. The value $\vec{v}$ determines where $q$ 's read-only transaction is serialized. In particular, for some $l, 0<l \leq k$, the read-only transaction of $q$ is serialized after $U_{l-1}$ and before $U_{l}$, and for each item $\imath_{f}$ in $\left\{\imath_{0} \ldots \imath_{t-1}\right\}$, either $v_{f}$ is zero and no update wrote to $\imath_{f}$ before $U_{l}$, or the last update to $\imath_{f}$ before $U_{l}$ wrote $v_{f}$ to $\imath_{f}$. Let $S$ be the serialization of execution $\widehat{E}_{k}$.

Since the read-only transactions executed by process $q^{\prime}$ are invisible, $\widehat{E}_{k}$ and $E_{k}$ are indistinguishable to $p_{0}, \ldots, p_{t-1}$ and $q$. Thus, they will execute the same steps in both executions. Note that $S$ is a serialization also for $E_{k}$. Since $S$ preserves the real-time order among transactions, $E_{k}$ is a flippable execution where the read-only transaction terminates and strict serializability is preserved, contradicting Lemma 1.

As discussed before the lemma, the existence of a flippable execution (guaranteed by Lemma 3) implies there is an augmented flippable execution, and hence, Lemma 9 implies the following impossibility result:

THEOREM 10. There is no weakly disjoint-access parallel STM implementation with invisible read-only transactions of a serializable $S T M$, in which read-only transactions always terminate successfully.

When a read-only transaction of $t \geq 2$ data items applies non-trivial primitives to at most $t-2$ base objects, the readonly transactions of $q^{\prime}$ in the augmented flippable execution are, in fact, invisible since their read set contains only two data items. As discussed before Lemma 9, the existence of a flippable execution (guaranteed by Lemma 6) implies there is an augmented flippable execution, and hence, Lemma 9 implies the following lower bound:

THEOREM 11. In a serializable disjoint-access parallel STM implementation for $t+2$ processes, where all read-only transactions terminate successfully, some read-only transaction of $t \geq 2$ data items applies non-trivial primitives to at least $t-1$ base objects.

Snapshot isolation [19, 23, 25] decouples the consistency of the reads and the writes, and guarantees a snapshot of the read set not older than the start of the transaction. The proof can be adapted to hold also when the consistency condition of the STM is snapshot isolation.

\section{RELATED WORK}

Many STM implementations are centralized; in particular, to determine a unique commit timestamp for transactions, the Lazy Snapshot Algorithm (LSA) [22] relies on a single shared monotonically increasing counter, while Transactional Locking II (TL2) [6] relies on a global clock. Both 
approaches introduce a single hot-spot accessed by all transactions, regardless of their data sets, and are therefore not disjoint-access parallel.

More recently, two STM implementations without a centralized hot-spot have been proposed. Avni and Shavit [4] present a thread-local clock mechanism that provides a decentralized solution for maintaining a consistent view. The key idea is using Lamport clock (scalar causal timestamps) instead of the real-time global clock. Integrated with TL2, this mechanism provides an STM supporting invisible readonly transactions, without a centralized contention point. A drawback of this algorithm is that transactions that terminated long before the current one may cause it to fail since the timestamp recorded for them is not current enough. Thus, read-only transactions are not wait-free. Imbs and Raynal [16] propose an opaque lock-based STM with no centralized hot-spot but their solution has visible reads.

Guerraoui and Kapalka [9] prove that obstruction-free implementations of software transactional memory cannot ensure strict disjoint-access parallelism. This property requires transactions with disjoint data sets not to access a common base object. This notion is stronger than the one originally proposed by Israeli and Rappoport [17], and commonly used in the literature [14], where two transactions with disjoint data sets are allowed to access the same base objects, provided they are connected via other transactions. All other transactions have to progress in parallel, even if they are concurrent. Their definition of strict disjoint-access parallelism, like our first definition (Definition 1), allows concurrent reads to the same base objects even by transactions that are not connected in the conflict graph.

Our lower bound applies to the notion of disjoint-access parallelism as originally defined in [17]. In contrast, the result of [9] does not hold for this weaker notion. Indeed, Herlihy et al. [13] present an obstruction-free and disjoint-access parallel STM. Obstruction-freedom does not prevent interfering concurrent processes from starving each other and thus, the implementation presented in [13] does not guarantee that a read-only transaction eventually terminates successfully.

Elsewhere, Guerraoui and Kapalka [10] prove a lower bound on the number of steps a process takes to successfully terminate a transaction, for every implementation that uses invisible reads, is single-version, and never aborts a transaction unless it conflicts with another live transaction. Our lower bound allows multi-version implementations, but requires read-only transactions to terminate successfully, regardless of overlapping transactions.

Serializability provides a weaker guarantee on the ordering of transactions (it does not have to respect the real-time order of non-overlapping ones). Nevertheless, our impossibility results hold also for serializable STMs that preserve the per-process order. Indeed, none of the serializable STM implementations presented in the literature, e.g. $[5,7,20$, 24], provides disjoint-access parallelism and wait-free, invisible read-only transactions. In fact, the impossibility results hold also for STMs that satisfy the even weaker condition of snapshot isolation known from the database literature [19, 25] and suggested as an efficient alternative to serializability for STMs [23].

Riegel et al. [24] proposed an STM implementation that supports invisible reads and is disjoint-access parallel, but it provides only causal serializability; moreover, read-only transactions may abort infinitely many times. Causal serializability is weaker than serializability since it allows different processes to have a different view of the system. This leaves open the question of whether our results holds for causally serializable STMs, or whether the algorithm of [24] can be extended to have wait-free read-only transactions.

A read-only transaction can be considered as a partial SCAN operation [3]: a partial snapshot objects is an atomic snapshot object [1], where processes can scan any subset of the components. In the wait-free algorithm for partial snapshot objects [3], scanners announce which components they are currently attempting to scan, i.e., read-only transactions are visible.

Our proof techniques draw ideas from the lower bounds on the step complexity of UPDATE operations in snapshot objects. Israeli and Shirazi [18] prove an $\Omega(m)$ lower bound on the number of steps to update a component in an $m$ component single-writer snapshot objects, implemented from single-writer registers. Attiya, Ellen and Fatourou [2] extend this lower bound to implementations of $m$-component multi-writer objects from base objects of any type.

\section{DISCUSSION}

This paper shows that no transactional memory implementation can be disjoint-access parallel and have invisible, wait-free read-only transactions. There are implementations that are disjoint-access parallel and have invisible but not wait-free read-only transactions [4, 13], while others have invisible, wait-free read-only transactions but are not disjointaccess parallel [22]. In principle, the invisibility of read-only transactions can also be sacrificed in order to keep them wait-free, and the implementation disjoint-access parallel. This can be done by treating the read set together with the write set and adapting a dynamic disjoint-access parallel implementation of multi-location synchronization operator, e.g., [12]. (This algorithm is not wait-free, but it seems that it can be made wait-free without sacrificing the other properties.) Thus, each of the assumptions made in our impossibility result is necessary, since removing either of them admits an implementation with the two remaining properties.

Our work joins recent efforts to explore the boundaries of STM implementations, so as to guide algorithm designers in their attempt to find better and more efficient implementations. Such boundaries demonstrate which directions are futile and which might lead to performance gains. It would be interesting to derive additional quantitative results on the complexity of transactions, and in particular, read-only transactions.

Our proof shows that the read-only transaction cannot terminate successfully, but it is possible to terminate it unsuccessfully, by aborting it; however, this abort is not justified by data conflicts. Moreover, when the read-only transaction is retried, it is possible to continue the construction and force it to abort again. An implementation is permissive with respect to a safety property [8] if it never aborts a transaction unless necessary for ensuring correctness. Our proof shows that a disjoint-access parallel implementation with invisible read-only transactions that always terminate - however, not always successfully - is not permissive with respect to opacity, strict serializability, serializability or snapshot isolation. We would like to further investigate the connections between our results and the study of 
unnecessary aborts $[7,8]$ or wasted work in STM implementations.

Acknowledgements. We would like to thank Rachid Guerraoui, Michal Kapalka and Martin Vechev for helpful comments.

\section{REFERENCES}

[1] Y. Afek, H. Attiya, D. Dolev, E. Gafni, M. Merritt, and N. Shavit. Atomic snapshots of shared memory. J. ACM, 40(4):873-890, 1993.

[2] H. Attiya, F. Ellen, and P. Fatourou. The complexity of updating multi-writer snapshot objects. In ICDCN '06, pages 319-330.

[3] H. Attiya, R. Guerraoui, and E. Ruppert. Partial snapshot objects. In SPAA '08, pages 336-343.

[4] H. Avni and N. Shavit. Maintaining consistent transactional states without a global clock. In SIROCCO '08, pages 131-140.

[5] U. Aydonat and T. Abdelrahman. Serializability of transactions in software transactional memory. In TRANSACT '08.

[6] D. Dice, O. Shalev, and N. Shavit. Transactional locking II. In DISC '06, pages 194-208.

[7] V. Gramoli, D. Harmanci, and P. Felber. Towards a theory of input acceptance for transactional memories. In OPODIS '08, pages 527-533.

[8] R. Guerraoui, T. A. Henzinger, and V. Singh. Permissiveness in transactional memories. In DISC '08.

[9] R. Guerraoui and M. Kapalka. On obstruction-free transactions. In SPAA '08, pages 304-313.

[10] R. Guerraoui and M. Kapalka. On the correctness of transactional memory. In PPoPP '08, pages 175-184.

[11] R. Guerraoui and M. Kapalka. The semantics of progress in lock-based transactional memory. In POPL '09, pages 404-415.

[12] T. L. Harris, K. Fraser, and I. A. Pratt. A practical multi-word compare-and-swap operation. In DISC '02, pages 265-279.
[13] M. Herlihy, V. Luchangco, M. Moir, and W. N. Scherer III. Software transactional memory for dynamic-sized data structures. In PODC '03, pages 92-101.

[14] M. Herlihy and N. Shavit. The Art of Multiprocessor Programming. Morgan Kaufmann, 2008.

[15] M. P. Herlihy and J. M. Wing. Linearizability: a correctness condition for concurrent objects. ACM Trans. Program. Lang. Syst., 12(3):463-492, 1990.

[16] D. Imbs and M. Raynal. A lock-based protocol for software transactional memory. In OPODIS '08, pages 226-245.

[17] A. Israeli and L. Rappoport. Disjoint-access-parallel implementations of strong shared memory primitives. PODC '94, pages 151-160.

[18] A. Israeli and A. Shirazi. The time complexity of updating snapshot memories. Inf. Process. Lett., 65(1):33-40, 1998.

[19] S. Lu, A. Bernstein, and P. Lewis. Correct execution of transactions at different isolation levels. IEEE Transactions on Knowledge and Data Engineering, 16(9):1070-1081, 2004.

[20] J. Napper and L. Alvisi. Lock-free serializable transactions. Technical Report TR-05-04, The University of Texas at Austin, 2005.

[21] C. H. Papadimitriou. The serializability of concurrent database updates. J. ACM, 26(4):631-653, 1979.

[22] T. Riegel, P. Felber, and C. Fetzer. A lazy snapshot algorithm with eager validation. In DISC '06, pages 284-298.

[23] T. Riegel, C. Fetzer, and P. Felber. Snapshot isolation for software transactional memory. In TRANSACT' 06 .

[24] T. Riegel, C. Fetzer, H. Sturzrehm, and P. Felber. From causal to z-linearizable transactional memory. In PODC '07, pages 340-341.

[25] G. Weikum and G. Vossen. Transactional Information Systems: Theory, Algorithms, and the Practice of Concurrency Control and Recovery. Morgan Kaufmann, 2001. 